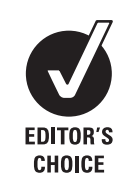

${ }^{1}$ Department of Occupational and Environmental Medicine, Graduate School of Medicine, Chiba University, Chiba, Japan; ${ }^{2}$ Center for Preventive Medical Science, Chiba University, Chiba, Japan; ${ }^{3}$ Division for Environment, Health and Safety, University of Tokyo, Tokyo, Japan

Correspondence to: Dr Yasushi Suwazono, Department of Occupational and Environmental Medicine (A2), Graduate School of Medicine, Chiba University, 1-8-1, Inohana, Chuoku, Chiba, 260-8670 Japan; suwa@faculty.chiba-u.jp

Accepted 17 October 2008

\title{
Shift work is a risk factor for increased total cholesterol level: a 14-year prospective cohort study in 6886 male workers
}

\author{
M Dochi, ${ }^{1}$ Y Suwazono, ${ }^{1,2}$ K Sakata, ${ }^{1}$ Y Okubo, ${ }^{3}$ M Oishi, ${ }^{1} \mathrm{~K}$ Tanaka, ${ }^{1}$ E Kobayashi, \\ K Nogawa'
}

\section{ABSTRACT}

Objectives: The widespread adoption of $24 \mathrm{~h}$ continuous operations in a number of industries has resulted in an increase in shift work, which may influence lipid metabolism because of disturbed circadian rhythms, broken sleep and lifestyle problems. The objective of the present study was to assess the effect of shift work on serum total cholesterol as an index of lipid metabolism.

Methods: A 14-year prospective cohort study was conducted in day workers ( $\mathrm{n}=4079)$ and alternating shift workers ( $n=2807$ ) who received annual health checkups between 1991 and 2005 in a Japanese steel company. The end-points were either a 20\%, 25\%, 30\%, $35 \%, 40 \%$ or $45 \%$ increase in serum total cholesterol during the period of observation, compared with serum total cholesterol at entry to the study. The association between the job schedule type and increase in serum total cholesterol was investigated using multivariate pooled logistic regression analyses. The odds ratios for the effect of shift work were obtained after adjustment for a number of potential confounders.

Results: The significant odds ratios of alternating shift work (and 95\% confidence intervals) were: $\geqslant 20 \%, 1.16$ (1.07 to 1.26$)$; $\geqslant 25 \%, 1.16$ (1.05 to 1.28$)$; $\geqslant 35 \%, 1.23$ (1.05 to 1.43$) ; \geqslant 40 \%, 1.30$ (1.07 to 1.58$)$; and $\geqslant 45 \%$, 1.28 (1.01 to 1.63) for serum total cholesterol.

Conclusion: Generally the odds ratios of alternating shift work tended to be higher for stricter cut-points of relative increase in serum total cholesterol level. Our study in male Japanese workers revealed that alternating shift work adversely affected lipid metabolism.

Industrialisation in Japan and other countries has led to the widespread adoption of $24 \mathrm{~h}$ continuous operations in a number of industries, including mining, manufacturing, transportation and servicetype companies. This has resulted in an increase in the proportion of the population routinely engaged in shift work. ${ }^{1}$ The Ministry of Health, Labour and Welfare reported that $22.7 \%$ of Japanese companies employed shift workers in $2005 .{ }^{1}$ The proportion of larger companies using shift workers has also increased, with $51.2 \%$ of companies with at least 1000 employees adopting a shift work schedule that includes fixed night work and alternating shift work. ${ }^{1}$ Although the effect of shift work on health has been studied extensively in other countries, a longitudinal investigation on the effect of shift work on the health of Japanese workers has not often been carried out and is therefore important. An association between shift work and cardiovascular disease has been reported. ${ }^{2-4}$ Disturbed

\section{What this paper adds}

- The effect of shift work on lipid metabolism has not often been assessed previously.

- In this study, alternating shift work was associated significantly with various endpoints for relative increase in serum total cholesterol.

- Generally the effects of alternating shift work tended to be larger for stricter endpoints.

- Efficient health screening and support to control unhealthy lifestyles may be of potential benefit in maintaining the health of alternating shift workers

circadian rhythms, broken sleep, lifestyle problems, and increased stress are thought to be potential mechanisms through which shift work increases the risk of cardiovascular disease. However, the link between cardiovascular disease and shift work is still questioned, and an investigation of lipid profiles, etc, among shift workers versus day workers will provide better understanding of how shift work might exert its effect.

Several studies have reported that elevated serum triglyceride $e^{5-7}$ and lower concentrations of high density lipoprotein cholesterol ${ }^{67}$ tend to occur more frequently in shift workers than in fixed daytime workers. In addition, several cohort studies $^{8}$ have reported an association between shift work and the risk of obesity or weight gain, suggesting a possible link between shift work and the development of the metabolic syndrome. Sleep debt is known to have a harmful impact on carbohydrate metabolism and endocrine function. ${ }^{10}$ It is therefore reasonable to expect that shift work may influence lipid metabolism, and that alternating between day shifts and night shifts, as occurs in alternating shift work, may be particularly deleterious to the health of workers. Some of the crosssectional and longitudinal studies on the relationship between shift work and hypercholesterolaemia, concluded that shift work may be a risk factor for hypercholesterolaemia, ${ }^{11-13}$ while others concluded that it was not. ${ }^{5}{ }^{14-16}$ However, most of the previous studies were cross-sectional and did not take into account confounding factors. Previously, we carried out a longitudinal study on the relationship between alternating shift work and the onset of hypercholesterolaemia in Japanese male workers, taking into account possible confounding 
factors. This study showed that shift work was a significant risk factor for the onset of hypercholesterolaemia, although the magnitude of the risk was small. ${ }^{17}$

As a consequence of those previous studies, a further longitudinal cohort study is needed to establish the degree to which shift work aggravates serum total cholesterol (T-Cho) level prior to the onset of hypercholesterolaemia. The object of the present study was to assess the effect of shift work on serum total cholesterol as an index of lipid metabolism. In the present study, the effect of shift work on increases in serum TCho was investigated after adjusting for baseline serum T-Cho level, age, body mass index (BMI), creatinine, glycated haemoglobin A1c (HbA1c), aspartate aminotransferase, $\gamma$ glutamyl transpeptidase (GGT), uric acid, drinking habit, smoking habit, and regular exercise using a multivariate pooled logistic regression model that took into account the effect of confounding influences and fluctuations in the variables.

\section{METHODS}

\section{Participants}

This prospective cohort study included observations on 8251 male workers in a Japanese steel company, collected over a 14year period from 1991 to 2005. The cohort consisted of more than $98 \%$ of the workers who attended annual health examinations during the observation period. New participants could be enrolled during the follow-up period. None of the subjects changed from alternating shift work to day shift work during the observation period due to an increase in T-Cho. Subjects treated previously for hypercholesterolaemia were excluded, while subjects who began pharmacological therapy for hypercholesterolaemia were classified as censored cases. The following individuals were excluded from this study: those undergoing a health examination for the first time in the final year (2005) of the follow-up period $(n=504)$, those who had pharmacological therapy initiated for hypercholesterolaemia before or in the year of entry $(n=46)$, those who had therapy initiated for hypercholesterolaemia in the subsequent year $(n=21)$, those with any missing data in the year of entry $(n=315)$, those who did not receive a health examination in the subsequent year $(n=360)$ and those for whom the measurement of T-Cho was missing in the subsequent year $(n=119)$. Thus, 1365 workers were excluded. A total of 6886 subjects were enrolled in the study, including 4079 day workers and 2807 alternating shift workers. The percentage of those dropping out of the workplace and the study ranged from $12.2 \%(\geqslant 20 \%$ increase in $\mathrm{T}$-Cho) to $17.2 \%$ ( $\geqslant 45 \%$ increase in $\mathrm{T}$-Cho) in each cohort for each end-point. The job schedule type (ie, shift work or day work) was determined from the payment ledger in May of each year. The shifts were scheduled on a four-team/threeshift plan with clockwise rotation ( 5 day shifts, 2 rest days, 5 evening shifts, 1 rest day, 5 night shifts and 2 rest days). The day, evening and night shifts started at 7:00 h, 15:00 h and 23:00 h, respectively. The study protocol was approved by the Ethics Review Board of the Graduate School of Medicine, Chiba University.

\section{Measurements}

The health examinations, including blood sampling, were carried out randomly between 9:00 $\mathrm{h}$ and 15:00 h throughout the study period. None of the measurements were taken within 30 min after a meal or heavy physical activity. The serum TCho of shift and day workers was measured by the cholesterol oxidase-peroxidase method (normal: $\leqslant 220 \mathrm{mg} / \mathrm{dl}(5.7 \mathrm{mmol} / \mathrm{l})$ ) after fasting or 30 or more minutes after a meal. The worker's medical history was recorded during the annual health examinations using a self-administered questionnaire. These responses were confirmed by individual interviews conducted by occupational physicians. The end-points in the study were defined as increases of either $20 \%, 25 \%, 30 \%, 35 \%, 40 \%$ or $45 \%$ in serum T-Cho level compared with baseline levels at entry to the study. These multiple cut-points allowed us to investigate the consistency and trend of results with more severe cutpoints. Age, BMI, creatinine, HbA1c, aspartate aminotransferase, GGT and uric acid were measured during the study, with smoking and drinking habits and regular exercise being used as covariates in the analyses. As far as possible, we chose the covariates from the available items investigated during the annual health examination, without overlapping with other measurements (such as aspartate and alanine aminotransferase for liver dysfunction) to avoid co-linearity in the logistic model. The laboratory tests were conducted in certified clinical testing laboratories. There was no change in the methods of laboratory tests over the study period which required conversion due to significant differences. Smoking and drinking habits and regular exercise were recorded at the annual health examinations using self-administered questionnaires. The self-administered questionnaire was developed at the beginning of the follow-up study with reference to previous studies by an occupational physician to encourage a healthy lifestyle in workers, based on their situation and medical needs in the workplace. ${ }^{18} 19$ The workers were asked to indicate whether they were a smoker or nonsmoker, drank alcohol every day or not, and exercised regularly or not. We did not ask about eating habits.

\section{Statistical analysis}

For the univariate analysis, the means of age, BMI, serum $\mathrm{T}$ Cho, creatinine, HbA1c, aspartate aminotransferase, GGT and uric acid at baseline were calculated. Differences in these variables between alternating shift and day workers were then evaluated using a Mann-Whitney U test. Baseline differences in smoking, drinking and exercise habits between the two groups were evaluated using the $\chi^{2}$ test. The incidence rates of each outcome per 1000 person-years were calculated, grouped according to job schedule type at entry. In the multivariate analysis, a pooled logistic regression analysis was used to evaluate the effect of alternating shift work on each of the six serum T-Cho end-points measured annually. All the covariates were included simultaneously in the statistical model. Using this method, the derived odds ratios (OR) for the end-points were adjusted for the effects of the other covariates. As the data on baseline serum T-Cho, creatinine, $\mathrm{HbA1c}$, aspartate aminotransferase, GGT and uric acid were not normally distributed, the values were logarithmically transformed using a base of 1.1. This transformation resulted in the odds ratio for the variables increasing by $10 \%$.

D'Agostino et a ${ }^{20}$ described pooled logistic regression analysis in detail. We consider a hypothetical study of 1000 persons at risk of a disease. All of these subjects have risk factors measured at time $t_{0}$ (or examination 1). We follow them through the first interval of observation. During that time period 40 subjects develop the disease and 10 others are lost to follow-up. We remove these 50 subjects from the population at risk. At time $t_{1}$ (examination 2) there are 950 subjects in whom risk factors are measured. Of these, 25 develop the disease and five are lost to follow-up. The remaining 920 subjects have risk factors measured at time $t_{2}$ (examination 3 ), of which 20 develop the disease in the next period and 10 are lost to follow-up. This 
method pools the subjects at risk in each interval to yield 2870 $(1000+950+920)$ person-examinations and pools the 85 $(40+25+20)$ disease events. A logistic regression with 2870 observations and 85 events constitutes a pooled logistic regression analysis.

Mathematically, the logistic regression model is written ${ }^{20}$ :

$$
\operatorname{logit} q_{i}\left(X\left(t_{i-1}\right)\right)=\log \left(\frac{q_{i}\left(X\left(t_{i-1}\right)\right)}{1-q_{i}\left(X\left(t_{i-1}\right)\right)}\right)=\alpha+\gamma_{1} X_{1}\left(t_{i-1}\right)+\ldots+\gamma_{p} X_{p}\left(t_{i-1}\right)
$$

where $q_{i}\left(X\left(t_{i-1}\right)\right)$ is the conditional probability of observing an event by time $t_{i}$ given that the individual is event-free at time $t_{i-1}$, and

$$
X\left(t_{i-1}\right)=\left(X_{1}\left(t_{i-1}\right), \ldots, X_{p}\left(t_{i-1}\right)\right)
$$

is the vector of risk factors measured at time $t_{i-1}$. The parameters obtained are adjusted for the effects of other timevarying covariates. Each examination interval of 1 year was treated as a mini follow-up study. Thus, this method included the concept of person-year. Moreover, the obtained odds ratio corresponds to the multivariate-adjusted incidence rate ratio of each outcome using the observed person-years. When the subject developed increased T-Cho during follow-up, subsequent data were excluded from the analyses. When there were missing data, subsequent data were similarly excluded. The analyses were performed with SPSS 15.0J software (SPSS, Tokyo, Japan). p Values $<0.05$ were considered to be statistically significant.

\section{RESULTS}

Table 1 summarises the baseline characteristics of the alternating shift workers and day workers at the year of entry into the study. BMI, HbA1c, creatinine and uric acid were significantly higher in day workers compared with alternating shift workers, while the alternating shift workers were significantly older and had higher aspartate aminotransferase. The percentage of subjects who drank or smoked every day or did exercise regularly was significantly higher in alternating shift workers than in day workers. The number of subjects and person-years studied are shown in table 2. Of the subjects in the cohort, $37.1 \%, 25.2 \%, 16.3 \%, 10.3 \%, 6.4 \%$ and $4.0 \%$ developed $\geqslant 20 \%$, $\geqslant 25 \%, \geqslant 30 \%, \geqslant 35 \%, \geqslant 40 \%$ and $\geqslant 45 \%$ increases in serum $\mathrm{T}$ Cho, respectively.

Table 3 shows the results of the pooled logistic regression analysis for increases in serum T-Cho. The ORs and 95\% confidence intervals ( $95 \% \mathrm{CIs}$ ) are grouped according to the serum T-Cho end-points. The type of job schedule was significantly associated with five serum $\mathrm{T}$-Cho end-points ( $\geqslant 20 \%$, OR 1.16 (95\% CI 1.07 to 1.26$) ; \geqslant 25 \%$, OR $1.16(95 \%$ CI 1.05 to 1.28$)$; $\geqslant 35 \%$, OR 1.23 (95\% CI 1.05 to 1.43$) ; \geqslant 40 \%$, OR 1.30 (95\% CI 1.07 to 1.58$)$; $\geqslant 45 \%$, OR 1.28 (95\% CI 1.01 to 1.63$)$ ). Generally the odds ratios of alternating shift work tend to be higher for stricter cut-points of relative increase in serum total cholesterol level. Multiple significant ORs were obtained for baseline T-Cho (negative: $\geqslant 20 \%, \geqslant 25 \%, \geqslant 30 \%, \geqslant 35 \%, \geqslant 40 \%$ and $\geqslant 45 \%$ ), BMI (positive: $\geqslant 25 \%, \geqslant 30 \%, \geqslant 35 \%, \geqslant 40 \%$ and $\geqslant 45 \%$ ), HbA1c (positive: $\geqslant 25 \%, \geqslant 30 \%, \geqslant 35 \%, \geqslant 40 \%$ and $\geqslant 45 \%$ ), GGT (positive: $\geqslant 20 \%, \geqslant 25 \%, \geqslant 30 \%, \geqslant 35 \%, \geqslant 40 \%$ and $\geqslant 45 \%$ ) and drinking habit (negative: $\geqslant 20 \%$ and $\geqslant 25 \%$ ). No consistent relationship was observed for age, creatinine, aspartate aminotransferase, uric acid, smoking or exercise habits.

\section{DISCUSSION}

The main finding of this 14-year, prospective, cohort study was that alternating shift work had a significant adverse effect on serum T-Cho level. Previous cross-sectional studies on the relationship between shift work and hypercholesterolaemia ${ }^{511-16}$ may have been influenced by some subjects ceasing shift work, resulting in retention of only healthy adaptable subjects in the shift worker groups. To overcome this bias, it is necessary to carry out a longitudinal study, similar to the present study, on a cohort of subjects over an extended period of time, while also taking into account health trends among those who drop out as far as possible. The participation rate was more than $98 \%$ in the present study. In addition, the pooled logistic regression analysis permits continuing follow-up of subjects who changed their job schedule type. Furthermore, increased T-Cho level was not related to change in job schedule type. In this company, an occupational physician was consulted and asked to approve all measures (ie, personnel relocation) taken to address the health problems of workers. Furthermore, the occupational physician

\begin{tabular}{|c|c|c|c|c|c|c|c|}
\hline & \multicolumn{7}{|c|}{ Job schedule type at entry year } \\
\hline & \multicolumn{2}{|l|}{ Day } & \multicolumn{2}{|l|}{ Shift } & \multicolumn{2}{|l|}{ Total } & \multirow[b]{2}{*}{ p Value } \\
\hline & Mean & SD & Mean & SD & Mean & SD & \\
\hline Total serum cholesterol (mg/dl) & 188.0 & 35.4 & 187.9 & 35.2 & 188.0 & 35.3 & 0.994 \\
\hline Age (years) & 36.2 & 10.7 & 36.9 & 9.4 & 36.5 & 10.2 & 0.003 \\
\hline Body mass index $\left(\mathrm{kg} / \mathrm{m}^{2}\right)$ & 23.5 & 2.9 & 23.3 & 3.0 & 23.4 & 2.9 & 0.001 \\
\hline Glycated haemoglobin A1c (\%) & 4.71 & 0.57 & 4.65 & 0.53 & 4.68 & 0.56 & $<0.001$ \\
\hline Creatinine (mg/dl) & 0.90 & 0.29 & 0.87 & 0.15 & 0.89 & 0.24 & $<0.001$ \\
\hline Aspartate aminotransferase (IU/I) & 22.2 & 17.7 & 22.4 & 10.4 & 22.3 & 15.2 & 0.003 \\
\hline$\gamma$-Glutamyl transpeptidase (IU/I) & 32.5 & 40.6 & 32.9 & 38.5 & 32.7 & 39.7 & 0.133 \\
\hline \multirow[t]{2}{*}{ Uric acid $(\mathrm{mg} / \mathrm{dl})$} & 5.68 & 1.21 & 5.61 & 1.26 & 5.65 & 1.23 & 0.009 \\
\hline & $\%$ & & $\%$ & & $\%$ & & p Value \\
\hline Drinking habit (everyday) & $37.4 \%$ & & $47.2 \%$ & & $41.4 \%$ & & $<0.001$ \\
\hline Smoking habit (smoker) & $57.6 \%$ & & $67.9 \%$ & & $61.8 \%$ & & $<0.001$ \\
\hline Regular exercise (absence) & $42.4 \%$ & & $46.8 \%$ & & $44.2 \%$ & & $<0.001$ \\
\hline No of subjects & 4079 & & 2807 & & 6886 & & \\
\hline
\end{tabular}

Table 1 Baseline characteristics of the subjects at their first health examination, grouped according to type of job schedule 
Table 2 The number of subjects and person-years studied according to type of job schedule at year of entry to the study

\begin{tabular}{|c|c|c|c|c|c|}
\hline Increase by & $\begin{array}{l}\text { Job schedule type at entry } \\
\text { year }\end{array}$ & $\begin{array}{l}\text { No of subjects whose total cholesterol } \\
\text { increased }(\mathrm{n}(\%))\end{array}$ & $\begin{array}{l}\text { Total person-years } \\
\text { of observation }\end{array}$ & $\begin{array}{l}\text { Incidence rate per } \\
1000 \text { person-years }\end{array}$ & $\begin{array}{l}\text { Mean observed years } \\
\text { per person }\end{array}$ \\
\hline \multirow[t]{2}{*}{$\geqslant 20 \%$} & Day & $1398(34.3)$ & 25060 & 55.8 & 6.1 \\
\hline & Shift & $1160(41.3)$ & 19488 & 59.5 & 6.9 \\
\hline \multirow[t]{3}{*}{$\geqslant 25 \%$} & Day & 931 (22.8) & 27542 & 33.8 & 6.8 \\
\hline & Shift & $802(28.6)$ & 21920 & 36.6 & 7.8 \\
\hline & Total & $1733(25.2)$ & 49462 & 35.0 & 7.2 \\
\hline$\geqslant 30 \%$ & Total & 1125 (16.3) & 52998 & 21.2 & 7.7 \\
\hline \multirow[t]{3}{*}{$\geqslant 35 \%$} & Day & $366(9.0)$ & 30468 & 12.0 & 7.5 \\
\hline & Shift & $340(12.1)$ & 24666 & 13.8 & 8.8 \\
\hline & Total & $706(10.3)$ & 55134 & 12.8 & 8.0 \\
\hline \multirow[t]{2}{*}{$\geqslant 40 \%$} & Day & $226(5.5)$ & 31030 & 7.3 & 7.6 \\
\hline & Shift & $212(7.6)$ & 25298 & 8.4 & 9.0 \\
\hline
\end{tabular}

interviewed the workers to determine their health status at the annual health examination. Therefore, we obtained considerable information indicating that workers changed jobs because of an increase in T-Cho level. There was only minimal loss of critical data and no inversion of cause and response. We therefore consider that retention bias was not likely to have affected the results of our study. A limitation regarding selection was that we could not evaluate differences in unmeasured background factors (including the history of job schedule type) between day and alternating shift workers.

As regards previous longitudinal studies, Morikawa et al followed up 1529 male blue-collar workers aged 19-49 years for 10 years. ${ }^{9}$ The participants were divided into four groups according to their work schedule at baseline and the end-point was workers doing fixed daytime work in both years (day-day, shift-day, day-shift and shift-shift). The relationships between

Table 3 Odds ratios and 95\% confidence intervals of shift work compared with day work for increases in total cholesterol

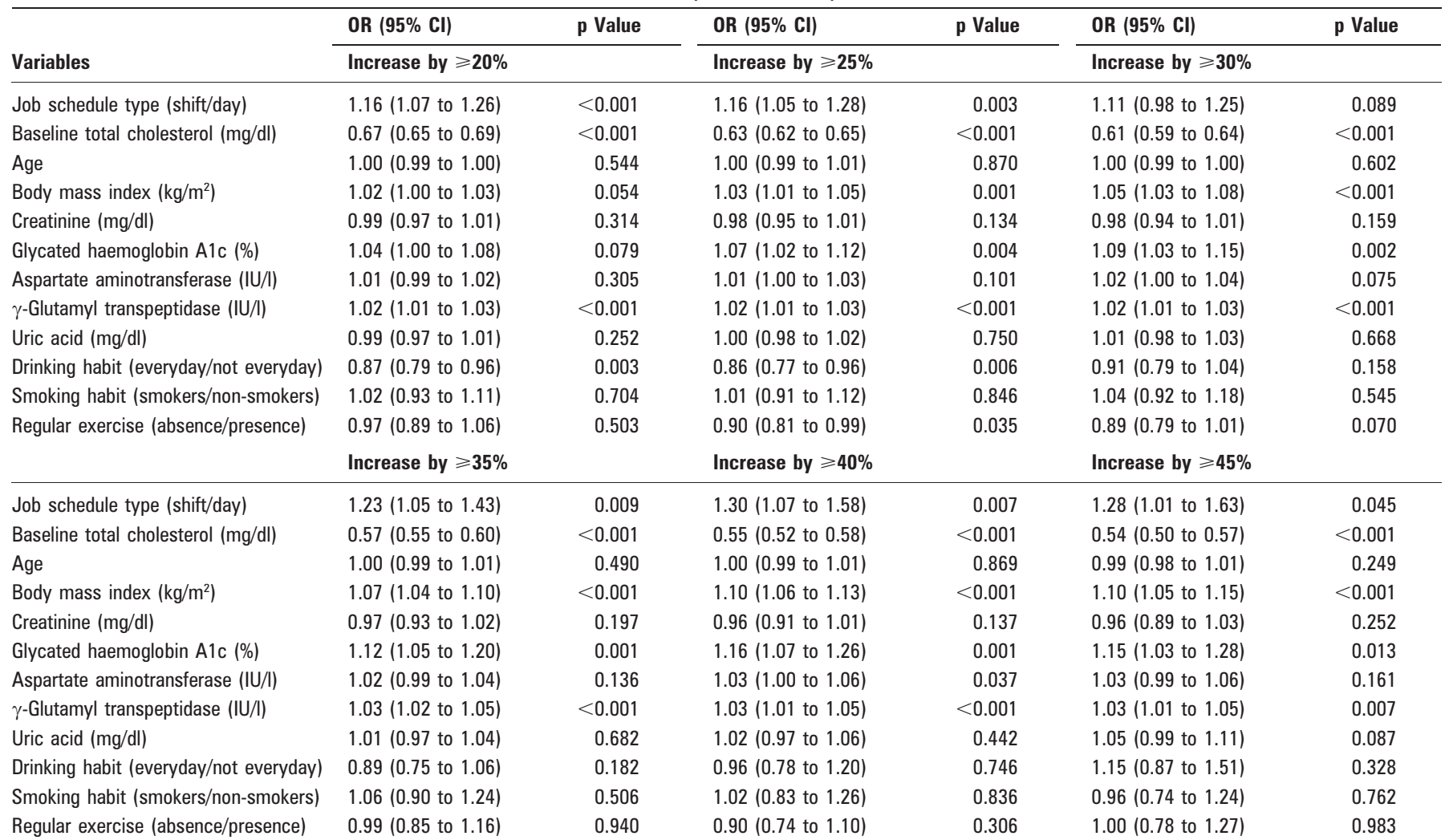

\footnotetext{
The data for total cholesterol, creatinine, glycated haemoglobin A1c, aspartate aminotransferase, $\gamma$-glutamyl transpeptidase and uric acid were logarithmically transformed using a base of 1.1. All covariates were included in the model.
}

$\mathrm{OR}$, odds ratio, estimated as the ratio of the former to the latter for job schedule type, drinking habits, smoking habits. 
changes in serum T-Cho during the 10-year period and working schedule were analysed adjusting for other confounding factors at baseline by using a multiple linear regression model. The authors did not find any significant difference in serum cholesterol levels between shift workers and day workers. However, one limitation of their study was that the fluctuation in other factors was not taken into consideration.

In contrast, we performed pooled logistic regression analysis in the present study. A strength of this multivariate method compared with other regression models is that the odds ratio is adjusted for variables such as job schedule type, BMI and lifestyle (data which are updated at each annual examination). In addition, pooled logistic regression analysis is equivalent to a Cox time-dependent regression analysis. ${ }^{20}$ This method of analysis has been used frequently in recent years. ${ }^{21-26}$

A further notable feature of our study was that the end-points were defined as either $20 \%, 25 \%, 30 \%, 35 \%, 40 \%$ or $45 \%$ increases in $\mathrm{T}$-Cho relative to the value measured at entry to the study. In our previous study, ${ }^{17}$ we found inconclusive results for the effect of shift work on hypercholesterolaemia using $\mathrm{T}$-Cho exceeding $220 \mathrm{mg} / \mathrm{dl}(5.7 \mathrm{mmol} / \mathrm{l})$ as the end-point. When a threshold value for T-Cho is used, some subjects will be excluded at the time of entry and this may introduce loss of information. Based on this experience, we reconsidered the study methods and adopted different end-points in the present study. These end-points allowed inclusion of more subjects regardless of $\mathrm{T}$-Cho level at baseline. Thus, we could obtain further information on the effect of alternating shift work on relative changes in T-Cho over time. On the other hand, we consider that using the end-points in the present study allowed us to detect a subclinical effect of alternating shift work on T-Cho, which was not detected by the clinical adverse response in previous study. ${ }^{17}$ From the viewpoint of preventive medicine, it is important to detect subclinical as well as clinical changes in T-Cho. Therefore, the results of the present study can be considered to complement the results of our previous study ${ }^{17}$ in establishing the effects of alternating shift work on T-Cho.

Three mechanisms have been implicated in the health effects of shift work. ${ }^{3}$ First, shift work influences the human body by interfering with the circadian rhythm. The second mechanism concerns behavioural changes including dietary habit, smoking habit and absence of regular exercise. Several studies ${ }^{27-29}$ have evaluated food intake and nutritional habits in shift workers. A comparison of different work shifts showed no significant variation in daily energy intake. ${ }^{27} 29$ The total number of eating events per day was found to be significantly higher in night shift workers, ${ }^{28}$ although these workers had a reduced energy intake during $8 \mathrm{~h}$ night shifts. ${ }^{29}$ In our study, we did not include the number of meals or daily food intake in the statistical model, as this information was not recorded at the beginning of the observation period. Therefore, it is possible that a preference for certain kinds of food will be another confounding factor for the increase in T-Cho. The third mechanism is social disruption, which makes it difficult for shift workers to spend time with their families, to contact their friends who are regular day workers and to fulfil the various social roles expected by society. This mechanism might cause stress. One study found that night workers had higher levels of catecholamine, a stress hormone, than day workers, ${ }^{13}$ while another showed that stress increased serum T-Cho. $^{30}$

A limitation of the present study was that we could not investigate other factors in detail, such as lifestyle, type of work, eating habits, and socioeconomic status during all follow-up periods. The simple questions on smoking, drinking and physical exercise did not allow us to adjust for differences in these habits or for unmeasured lifestyle choices such as eating habits. On the other hand, BMI and other biological characteristics were included in the model as covariates. Assessment of these items at the annual health examination as objective markers for various lifestyle related diseases was required by law. These items also reflect the effects of various lifestyles, especially in healthy subjects. Thus, we consider BMI and data on various other biological characteristics could complement our data on self-reported factors related to lifestyle.

In relation to the type of job, Bøggild et $a^{\beta 1}$ found that, in a random sample of Danish employees, shift workers report higher exposure to an unfavourable work environment compared with day workers, which may act as a confounder. However, because all subjects in the present study were employed by a large company in Japan, their working environments were very similar. The efforts of employers in such companies to protect workers from harmful work environments generally exceed legal requirements. In addition, we administered an extensive questionnaire in $2002^{32}$ which showed that $90 \%$ of alternating shift workers and $40 \%$ of day workers engaged in onsite work. Of the day workers, $20 \%$ were engaged in office work and $22 \%$ in research and technical work. In the company we studied, onsite workers are engaged in activities related to steel production and equipment maintenance and usually monitor and operate the production process remotely in a safe and comfortable operations room, without the demand for heavy physical labour. Occasionally these workers enter the production site to carry out equipment maintenance while the production process is suspended. We therefore consider work type was not a major confounding factor in our study. We could not include "psychological demands" as a confounder because a validated questionnaire in Japanese was not available at the beginning of this longitudinal study. On the basis of the results obtained in 2002,, alternating shift work was related to low job control but not to psychological and physiological demands. In the present study, low job control might have to be taken into consideration.

As for socioeconomic status, marital status and living arrangements did not differ between day workers and shift workers. For example, $79 \%$ of day workers were married compared with $74 \%$ of alternating shift workers, while $87 \%$ of day workers lived with their family compared with $86 \%$ of alternating shift workers. ${ }^{32}$ The economic status of the shift workers was generally good as they received a shift allowance. Educational levels were very similar between the day and alternating shift workers, with the majority of workers being high school graduates. Very few university graduates were engaged in daytime work. Therefore, we consider that socioeconomic status was not a major confounding factor in the present study.

On the other hand, shift workers were older than day workers at baseline, which was surprising as it might be expected that older people would refrain from shift work. Although it is difficult to identify the reasons for this result, we consider that older workers perform shift work in this company because they receive good health care and are subject to good labour management practices.

In conclusion, our study in male Japanese workers revealed that alternating shift work is an independent risk factor for deterioration in lipid metabolism. Generally, the odds ratios of alternating shift work tend to be higher for stricter cut-points of relative increase in serum total cholesterol level. The studied company had an alternating shift work schedule common in Japanese factories. Efficient health screening and regular check ups, combined with support to control unhealthy lifestyle 
habits, therefore, have the potential to be of considerable benefit in maintaining the health of shift workers.

Funding: This study was supported by a grant from the Japan Society for the Promotion of Science (Grants-in-Aid for Scientific Research, (C) no. 17590508). The funding source had no involvement in study design; in the collection, analysis, and interpretation of data; in the writing of the report; and in the decision to submit the paper for publication.

Competing interests: None.

Ethics approval: The study protocol was approved by the Ethics Review Board of the Graduate School of Medicine, Chiba University.

Provenance and peer review: Not commissioned; externally peer reviewed.

\section{REFERENCES}

1. Ministry of Health, Labour and Welfare, Japan. General survey on working conditions in 2005 (in Japanese). Tokyo: Ministry of Health, Labour and Welfare, 2005.

2. Kristensen TS. Cardiovascular diseases and the work environment. A critical review of the epidemiologic literature on nonchemical factors. Scand J Work Environ Health 1989:15:165-79.

3. Knutsson A, Bøggild H. Shiftwork and cardiovascular disease: review of disease mechanisms. Rev Environ Health 2000;15:359-72.

4. Bøggild H, Knutsson A. Shift work, risk factors and cardiovascular disease. Scand J Work Environ Health 1999;25:85-99.

5. Knutsson A, Akerstedt T, Jonsson BG. Prevalence of risk factors for coronary artery disease among day and shift workers. Scand J Work Environ Health 1988;14:317-21.

6. Karlsson B, Knutsson A, Lindahl B. Is there an association between shift work and having a metabolic syndrome? Results from a population based study of 27,485 people. Occup Environ Med 2001;58:747-52.

7. Karlsson BH, Knutsson AK, Lindahl BO, et al. Metabolic disturbances in male workers with rotating three-shift work. Results of the WOLF study. Int Arch Occup Environ Health 2003;76:424-30.

8. Niedhammer I, Lert F, Marne MJ. Prevalence of overweight and weight gain in relation to night work in a nurses' cohort. Int J Obes Relat Metab Disord 1996:20:625-33.

9. Morikawa Y, Nakagawa H, Miura K, et al. Effect of shift work on body mass index and metabolic parameters. Scand J Work Environ Health 2007:33:45-50.

10. Spiegel K, Leproult R, Van Cauter E. Impact of sleep debt on metabolic and endocrine function. Lancet 1999;354:1435-9.

11. Ghiasvand $\mathbf{M}$, Heshmat R, Golpira R, et al. Shift working and risk of lipid disorders: a cross-sectional study. Lipids Health Dis 2006;5:9.

12. Ha M, Park J. Shiftwork and metabolic risk factors of cardiovascular disease. J Occup Health 2005:47:89-95.

13. Theorell T, Akerstedt T. Day and night work: changes in cholesterol, uric acid, glucose and potassium in serum and in circadian patterns of urinary catecholamine excretion. A longitudinal cross-over study of railway workers. Acta Med Scand 1976;200:47-53.
14. Bursey RG. A cardiovascular study of shift workers with respect to coronary artery disease risk factor prevalence. J Soc Occup Med 1990;40:65-7.

15. Romon M, Nuttens MC, Fievet $\mathrm{C}$, et al. Increased triglyceride levels in shift workers Am J Med 1992;93:259-62.

16. Tenkanen L, Sjöblom T, Kalimo R, et al. Shift work, occupation and coronary heart disease over 6 years of follow-up in the Helsinki Heart Study. Scand J Work Environ Health 1997;23:257-65.

17. Dochi M, Sakata K, Oishi M, et al. Relationship between shift work and hypercholesterolemia in Japan. Scand J Work Environ Health 2008;34:33-9.

18. Belloc NB, Breslow L. Relationship of physical health status and health practices Prev Med 1972;1:409-21.

19. Hagihara A, Morimoto K. Personal health practices and attitudes toward nonsmokers' legal rights in Japan. Soc Sci Med 1991:33:717-21.

20. D'Agostino RB, Lee ML, Belanger AJ, et al. Relation of pooled logistic regression to time dependent Cox regression analysis: the Framingham Heart Study. Stat Med 1990;9:1501-15.

21. Schett G, Kiechl S, Redlich K, et al. Soluble RANKL and risk of nontraumatic fracture. JAMA 2004:291:1108-13.

22. Oishi M, Suwazono Y, Sakata K, et al. A longitudinal study on the relationship between shift work and the progression of hypertension in male Japanese workers. J Hypertens 2005;23:2173-8.

23. Suwazono $\mathbf{Y}$, Kobayashi $\mathrm{E}$, Uetani $\mathrm{M}$, et al. The $-1438 \mathrm{~A} / \mathrm{G}$ polymorphism in the 5 hydroxytryptamine receptor $2 \mathrm{~A}$ gene is related to hyperuricemia, increased gammaglutamyl transpeptidase and decreased high-density lipoprotein cholesterol level in the Japanese population: a prospective cohort study over 5 years. Int J Mol Med 2006;17:77-82.

24. Sakata K, Suwazono Y, Harada H, et al. The relationship between shift work and the onset of hypertension in male Japanese workers. J Occup Environ Med 2003:45:1002-6.

25. Fairfield KM, Willett WC, Rosner BA, et al. Obesity, weight gain, and ovarian cancer. Obstet Gynecol 2002;100:288-96.

26. Suwazono Y, Okubo Y, Kobayashi E, et al. A follow-up study on the association of working conditions and lifestyles with the development of (perceived) mental symptoms in workers of a telecommunication enterprise. Occup Med (Lond) 2003; 53:436-42.

27. de Assis MA, Kupek E, Nahas MV, et al. Food intake and circadian rhythms in shift workers with a high workload. Appetite 2003;40:175-83.

28. de Assis MA, Nahas MV, Bellisle F, et al. Meals, snacks and food choices in Brazilian shift workers with high energy expenditure. J Hum Nutr Diet 2003;16:283-9.

29. Lennernäs M, Hambraeus L, Ảkerstedt T. Shift related dietary intake in day and shift workers. Appetite 1995;25:253-65.

30. Clark DA, Arnold EL, Foulds EL Jr, et al. Serum urate and cholesterol levels in Air Force Academy cadets. Aviat Space Environ Med 1975;46:1044-8.

31. Bøggild H, Burr $\mathrm{H}$, Tüchsen $\mathrm{F}$, et al. Work environment of Danish shift and day workers. Scand J Work Environ Health 2001:27:97-105

32. Harada H, Suwazono Y, Sakata K, et al. Three-shift system increases job-related stress in Japanese workers. J Occup Health 2005;47:397-404. 